\title{
Estudis epidemiològics a la Comunitat Valenciana: el projecte ESCARVAL (2008-2019)
}

\author{
Domingo Orozco (dorozco@umh.es) \\ Universitat Miguel Hernández \\ Vicente Pallarés (pallarev@uji.es) \\ Universitat Jaume I \\ Vicente Gil GuiLlÉn (vgil@umh.es) \\ Universitat Miguel Hernández
}

\section{Introducció}

\subsection{Malaltia cardiovascular}

Les malalties del sistema circulatori constitueixen la primera causa de mort per al conjunt de la població espanyola. Segons dades de l'Institut Nacional d'Estadística, l'any 2012 el grup de les malalties del sistema circulatori es va mantenir com la primera causa de mort (responsables de 30,3 de cada 100 defuncions; INE 2012, SEH 2005). Per sexe, les malalties del sistema circulatori van ser la primera causa de mortalitat femenina (282,2 morts per cada 100.000) i la segona entre els homes (239,4 morts per cada 100.000). Si es comparen les taxes de mortalitat d'Espanya ajustades per edat amb les d'altres països occidentals, s'observa que pel que fa al total de les malalties de l'aparell circulatori i a la malaltia isquèmica del cor, Espanya té unes taxes relativament més baixes. Quant a la mortalitat per malaltia cerebrovascular, hi ocupa una posició intermèdia-baixa (Menotti i altres, 2000).

Es preveu un augment en el nombre d'hospitalitzacions per aquestes malalties en els pròxims anys com a conseqüència: del desenvolupament tecnològic que permetrà oferir als pacients nous instruments diagnòstics i terapèutics, de la major supervivència dels pacients amb aquests problemes de salut i, en menor grau, de l'envelliment de la població espanyola. Per tant, el nombre de malalts i de recursos sanitaris utilitzats actualment s'incrementarà en els pròxims anys a causa del creixement de la població envellida.

\subsection{Factors de risc clàssics i nous}

L'article publicat per Banegas i altres (2003) va analitzar la càrrega actual de la mortalitat atribuïble a alguns dels principals factors de risc cardiovascular (FRCV) en adults a Espanya. A prop de 14.000 morts totals (25\% de totes les morts) van ser atribuïbles a la hipertensió arterial (HTA) en la població de mitjana edat d'Espanya.

Una quarta part de les cuals van ser morts cardiovasculars; vora 56.000 morts eren atribuïbles al consum de tabac en adults ( $\geq 35$ anys) (16\% de totes les morts); per cardiopatia 
isquèmica el $12,8 \%$ i per accident cerebrovascular el 9,2\%. Gairebé 28.000 morts $(8,5 \%$ de totes les morts) es van produir per l'excés de pes en adults, dos terços dels quals eren morts cardiovasculars. Sobre 2.800 morts cardiovasculars en adults ( $\geq 35$ anys) eren atribuïbles a la diabetis (6\% de totes les morts cardiovasculars). En un altre estudi publicat per Medrano i altres (2007) ens parlen sobre la incidència ajustada de malaltia coronària atribuïble al sobrepès, el 42,5\% (6,8-59,6); al consum de tabac, el 33,9\% (22,6-41,0); a la hipercolesterolèmia el 19,4\% (8,2-26,5); i el 15,5\% (1,6-24,6) a la HTA. Entre les dones, el $36,5 \%(8,0-56,3)$ dels casos de cardiopatia isquèmica es van atribuir al sobrepès, el 24,8\% $(12,0-31,9)$ a la diabetis, i el 20,1\% (6,1-28,6) a la hipercolesterolèmia.

El descobriment i modificació d'aquests factors de risc clàssics (HTA, dislipèmia, tabaquisme, diabetis, inactivitat física i dieta occidental) ha permès reduir la mortalitat cardiovascular en els països desenvolupats (Folsom, 2013). Recentment, Lloy-Jones i altres (2010) conclouen que entre el 75\% i el 85\% de les malalties isquèmiques del cor als Estats Units es pot prevenir evitant aquests factors de risc clàssics. Respecte als nous factors de risc, s'han publicat múltiples estudis en què s'han identificat nous marcadors o factors, però en una recent revisió de Folsom (2013) es conclou que, a pesar de l'entusiasme per identificar nous factors, molt pocs s'han acceptat per al seu ús en la pràctica clínica. L'American College of Cardiology (ACC) i l'American Heart Association (AHA), en un grup conjunt de treball (Greenland i altres, 2010), recomanen l'ús dels antecedents familiars, l'hemoglobina glicosilada A1c, i la microalbuminúria (en pacients amb HTA o diabetis).

Pel que fa a altres factors, la proteïna $\mathrm{C}$ reactiva, la lipoproteïna associada fosfolipasa $\mathrm{A}_{2}$, el calci coronari, el gruix de la capa íntima-mitjana de la caròtida i l'índex turmell braç podrien ser utilitzats raonablement com a complement dels FRCV clàssics en individus classificats de risc intermedi i que poden reclassificar aquests pacients en un risc superior. No recomanen, de moment, l'ús de pèptids natriurètics, apolipoproteïnes o marcadors genètics. No obstant això, l'enorme coneixement existent dels FRCV clàssics no ha possibilitat el seu control en uns nivells acceptables. A Espanya, la importància epidemiològica i clínica dels FRCV clàssics com ara la HTA, la dislipèmia i la diabetis es descriu en l'estudi ENRICA (Banegas i altres, 2011).

El 33\% de la població espanyola presenta HTA, el 50,3\% presenta dislipèmia i el $6,9 \%$ diabetis mellitus. Menys del 50\% de les persones amb HTA que reben tractament presenten bon control. Si es té en compte que al voltant del $40 \%$ no estan diagnosticats i que, dels diagnosticats, un $20 \%$ no rep tractament, la proporció de pacients controlats és del $21,6 \%$, és a dir, només un de cada cinc. La hipercolesterolèmia és el factor de risc amb més prevalença i afecta el $50 \%$ de la població espanyola; no obstant això, és el menys conegut (53\%) i el menys tractat $(44,1 \%)$.

A més a més, dels tractats, només presenta bon control el 55,7\% (Guallar-Castillón i altres, 2012). Tenint en compte aquestes dades, la proporció de pacients controlats sobre el total de pacients amb dislipèmia és del 13,2\%, és a dir, només un de cada deu. El cas de la diabetis és el més favorable, perquè és el més conegut (79\%), el més tractat (88\%) i, dels tractats, el que presenta el millor control (69\%). Tanmateix, un altre estudi recent (Soriguer, 2012), que empra sobrecàrrega oral de glucosa com a mètode de diagnòstic, descriu una prevalença de $13,8 \%$, amb un $6 \%$ de diabetis no coneguda prèviament, i un $66 \%$ de pacients tractats farmacològicament (Rojo-Martínez, 2013). 


\subsection{Escales de risc}

La malaltia cardiovascular (MCV) es caracteritza, per tant, per tenir una etiologia multifactorial, atès que els FRCV associats es potencien entre si. Per això, l'abordatge correcte de la prevenció cardiovascular requereix una valoració conjunta d'aquests FRCV (Rose, 1985). Així, l'AHA recomana que, per a la prevenció de les MCV, s'han de mesurar els factors de risc i s'ha de calcular el risc global de malaltia coronària en tots els pacients adults (Greenland, 2010). Però es recomana no sols la prevenció primària sinó també la prevenció primordial. El risc cardiovascular (probabilitat de patir una malaltia coronària i cerebrovascular, a diferència del risc coronari de patir només una malaltia coronària), estableix la probabilitat de patir una $\mathrm{MCV}$ en un determinat període de temps, generalment deu anys.

L'estratificació del risc cardiovascular mitjançant escales és un pilar central per a prendre decisions terapèutiques en prevenció cardiovascular. Les escales derivades de l'estudi Framingham (als EUA), que calculen la morbimortalitat coronària (Wilson, 1998), i les del projecte Score (a Europa), que calculen la mortalitat cardiovascular (Conroy, 2003), en ambdós casos a deu anys, són les de més difusió en pràctica clínica. Distintes anàlisis conclouen, no obstant això, que Framingham-Wilson sobreestima el risc coronari en països del sud d'Europa, on la incidència d'infart agut de miocardi és menor (Menotti i altres, 2000, Masiá i altres, 1998). Per això, distintes societats científiques suggereixen realitzar estudis de cohorts poblacionals en àrees concretes, per a obtenir escales pròpies o adaptar les existents (Menotti i altres, 2000, D’Agostino i altres, 2001, Hense i altres, 2003).

\subsection{Calibratge d'escales}

A Espanya, s'ha calibrat l'equació de Framingham-Wilson (Wilson i altres, 1998) en la població de Girona amb una metodologia validada (D'Agostino i altres, 2001), i s'ha obtingut l'escala de Regicor (Marrugat i altres, 2003). Mitjançant dissenys transversals, s'han obtingut també les taules DORICA (dislipèmia, obesitat i risc cardiovascular, Aranceta i altres, 2004). D'altra banda, després de la publicació dels resultats del projecte Score, on Espanya va participar amb tres cohorts (Conroy i altres, 2003), diverses societats (informe de les societats europees d'arteriosclerosi, Comitè Espanyol Interdisciplinari per a la Prevenció Cardiovascular, CEIPC, i Programa d'Activitats Preventives i Promoció de la Salut de la Societat Espanyola de Medicina Familiar i Comunitària, PAPPS, De Backer i altres, 2003, Brotons i altres, 2004), recomanen utilitzar l'escala Score en la seua versió per a països de baix risc per al càlcul de la mortalitat cardiovascular al nostre país.

Totes les societats científiques espanyoles han protocol-litzat aquesta escala per a població general i és la que s'utilitza per al seguiment de pacients hipertensos, dislipèmics i diabètics, però presenta un gran inconvenient en predir només mortalitat cardiovascular i no morbiditat (possibilitat d'emmalaltir), amb la qual cosa l'edat del pacient té un pes específic molt important i possiblement se subestime el risc en població més jove i amb FRCV. A més, aquestes escales de risc s'han construït en població natural i no són el resultat de seguiment de pacients amb una patologia determinada, com ara hipertensos, dislipèmics o diabètics. Els clínics coneixedors d'aquesta altra limitació la qüestionen i en condicionen l'ús en la pràctica mèdica. 
Hi ha gran variabilitat en la pràctica clínica, generada per la valoració del pronòstic d'aquests pacients, a causa de les diferents recomanacions en l'ús d'escales de risc cardiovascular, segons s'utilitzen unes guies clíniques o altres.

\subsection{La importància de la història clínica electrònica}

Els registres electrònics longitudinals de salut presenten noves possibilitats per a la millor comprensió de la prestació de l'atenció sanitària i l'avaluació dels seus resultats (Maiques, 2014). Aquests registres poden permetre respondre a moltes preguntes ara sense resposta que han d'abordar-se en relació amb les mesures de prevenció, proves de diagnòstic i tractaments. Ofereixen noves oportunitats per als investigadors cardiovasculars que busquen frenar aquesta principal causa de mort i discapacitat.

La història clínica electrònica (HCE) és una nova oportunitat en investigació cardiovascular per diversos motius: $a$ ) el personal mèdic i altres professionals clínics han d'entendre que l'HCE no sols permet una major comunicació amb els pacients i altres professionals de la salut, sinó que també facilita l'anàlisi conjunta per a avaluar millor l'atenció prestada; $b$ ) estratificar el nivell de risc dels pacients podria alleujar les càrregues administratives i els retards i centrar els esforços en protocols on la supervisió intensiva és més beneficiosa; i $c$ ) per a les malalties a llarg termini, com ara la insuficiència cardíaca, la fibrillació auricular, la hipertensió i la hiperlipidèmia, és important integrar les dades de registre amb les dades de l'HCE i la creixent varietat de mesuraments de resultat informats pels pacients.

Experiències inicials a Suècia (TASTE) (Califf, 2013) i als Estats Units (SAFE-PCI, Hess, 2013) demostren que fins i tot els assajos aleatoris es poden realitzar a través de l'HCE usual amb un cost molt menor al dels assajos tradicionals. Els pacients amb malalties cròniques o FRCV com ara HTA, DM o DLP, sovint treballen amb molts professionals de la salut (metges, infermeres i altres especialistes). Per assegurar una transició adequada d'un lloc a un altre, els professionals de salut han de compartir informació i coordinar l'atenció amb eficàcia (Maiques i altres, 2014).

Els registres mèdics electrònics (eTools) s'estan utilitzant cada vegada més per a coordinar l'atenció al pacient. Hi ha proves recents (HQO, 2013) que determinen que les eines electròniques de registre adequades, en l'entorn i context adequats, poden millorar significativament la utilització dels serveis de salut. Al Regne Unit, s'ha realitzat recentment una validació de l'escala de risc QRISK2-2011 amb la utilització d'una cohort prospectiva de dades poblacionals a través de la base de dades The Health Improvement Network (THET) (Collins i Altman, 2012).

L'existència d'una HCE ambulatòria única integrada a la Comunitat Valenciana garanteix la viabilitat d'aquest tipus d'investigació analítica poblacional i permetrà donar resposta a moltes preguntes clíniques que romanen sense resoldre, ja que facilitarà i farà efectiva la presa de decisions mèdiques. L'HCE valenciana es va gestar el 2001 i té com a objectiu generar una història clínica única d'accés per a tot el sistema públic sanitari valencià. Actualment cobreix més del 95\% de la població total de la Comunitat Valenciana (5,2 milions d'habitants).

A hores d'ara, integra tota l'assistència ambulatòria (primària i especialitzada), i ofereix accés a l'episodi d'ingrés hospitalari, encara que durant aquest s'empra un altre 
sistema informàtic variable entre hospitals. En qualsevol cas, s'hi pot accedir al full d'alta amb els diagnòstics i els tractaments a través del sistema CMBD. Engloba un mòdul administratiu per a gestionar, per exemple, la citació del pacient i un mòdul assistencial que ens permet conèixer la informació sanitària en temps real. Per evitar el biaix d'infraregistre, és fonamental una formació continuada sobre el funcionament i emplenament de l'HCE destinada als professionals, com ja s'ha realitzat en el projecte Escarval.

\section{Hipòtesi i objectius del projecte Escarval}

\subsection{Hipòtesi}

L'elaboració d'escales pròpies de risc cardiovascular poblacional permet millorar la predicció del risc ajustat per a població amb HTA, diabetis i/o dislipèmia de la Comunitat Valenciana. De la mateixa manera, l'estudi de factors no tradicionals pot oferir una precisió més elevada en la determinació del risc per a millorar en el nivell d'intervenció i en la protecció del risc cardiovascular i renal.

\subsection{Objectius}

L'objectiu de l'estudi Escarval Risc és generar i validar una escala predictiva de risc cardiovascular en pacients majors de 40 anys, diagnosticats d'HTA, dislipèmia o diabetis mellitus.

Fins a l'actualitat s'ha finalitzat un seguiment inicial de cinc anys (2008-2012) sobre 50.000 pacients basat en el registre electrònic de la història de salut. La informació es registra i es recull a través de l'HCE. Es tracta d'un disseny observacional, longitudinal, de cohorts i prospectiu en l'àmbit de la Comunitat Valenciana.

\section{Situació del projecte en el moment actual}

El projecte Escarval és un programa que inclou no sols una línia d'investigació sinó també una línia de formació continuada i una altra de millora de la pràctica clínica.

S'han realitzat tres cursos de formació continuada en línia en patologia cardiovascular a través de l'Escola Valenciana d'Estudis per a la Salut (EVES) amb la participació de més de mil professionals en cada curs. S'han publicat diverses monografies clíniques sobre la situació de la patologia cardiovascular a la Comunitat Valenciana.

El projecte ha servit també per a millorar el full de seguiment cardiovascular de l'HCE Abucasis, així com per a promoure la implantació del laboratori en línia que permet rebre els valors de les analítiques directament del laboratori, amb la supervisió i l'aprovació de l'analista, sense necessitat de transcripció manual a l'HCE amb la font d'error que això pot suposar tant des del punt de vista qualitatiu (no es transcriuen tots els valors) com quantitatiu (en el valor de les xifres).

El projecte Escarval s'estructura en tres grans projectes d'investigació amb una duració d'onze anys (2008-2019): 
- Escarval Transversal. El seu objectiu és descriure, des de 2008, la situació del mapa cardiovascular de la Comunitat Valenciana i la seua evolució al llarg dels anys a través de la realització de talls transversals de la informació registrada sobre patologia cardiovascular en l'HCE Abucasis de la població de la Comunitat Valenciana i la seua evolució anual. Inclou tant els esdeveniments cardiovasculars com la situació dels FRCV quant a prevalença i control.

De l'anàlisi de la situació transversal cardiovascular, es pot concloure un augment important després de la formació en línia de la prevalença dels FRCV a la Comunitat Valenciana amb un millor control d'aquests i una reducció del seu desconeixement. Això ha sigut a causa del millor registre dels FRCV en l'HCE, més integració de l'activitat preventiva en la pràctica clínica, millor compliment per part dels pacients dels seus tractaments i reducció de la inèrcia clínica.

- Escarval Risc. El seu objectiu és conèixer la incidència dels esdeveniments CV i els FRCV associats mitjançant anàlisi de la informació registrada sobre patologia cardiovascular en l'HCE Abucasis de la població amb HTA, diabetis i/o dislipèmia de la Comunitat Valenciana. Fou inicialment previst per a cinc anys (2008-2012) i posteriorment ampliat fins a l'any 2019. Inclou la construcció d'una escala de risc $\mathrm{CV}$ per a la població de risc (HTA, diabetis i/o dislipèmia) de la Comunitat Valenciana. Aquest és el projecte que es presenta en aquest capítol.

En el moment actual s'han generat nomogrames per al càlcul del risc cardiovascular amb dades pròpies. Es coneixen tant els riscs relatius com els riscs absoluts dels FRCV en la incidència d'esdeveniments cardiovasculars i han sigut cridaners els resultats que s'han trobat respecte al colesterol anomenat bo (c-HDL), l'hemoglobina glicosilada i els paràmetres de funció renal.

- Escarval Prevenció. El seu objectiu és conèixer la incidència d'esdeveniments CV i FRCV associats mitjançant anàlisi de la informació registrada sobre patologia cardiovascular en l'HCE Abucasis de la població de la Comunitat Valenciana. Inclou la construcció d'una escala de risc CV per a la població general de la Comunitat Valenciana. En el moment actual s'està finalitzant la inclusió de pacients.

En l'esquema següent, s'integren els tres projectes d'investigació prèviament comentats. Per a l'any 2020, els investigadors de l'estudi esperem obtenir una escala de risc amb dades pròpies per a ser aplicable a la Comunitat Valenciana que ens ajudarà a reduir la mortalitat cardiovascular, ja que a través d'aquesta funció de risc podem identificar individus amb risc cardiovascular alt $i$, a través de mesures higienicodietètiques i/o farmacològiques, podrem reduir la probabilitat que aquests individus patisquen l'esdeveniment cardiovascular. Igual que els nomogrames obtinguts l'any 2015, totes aquestes escales seran de fàcil implementació en la pràctica clínica de l'atenció primària. 


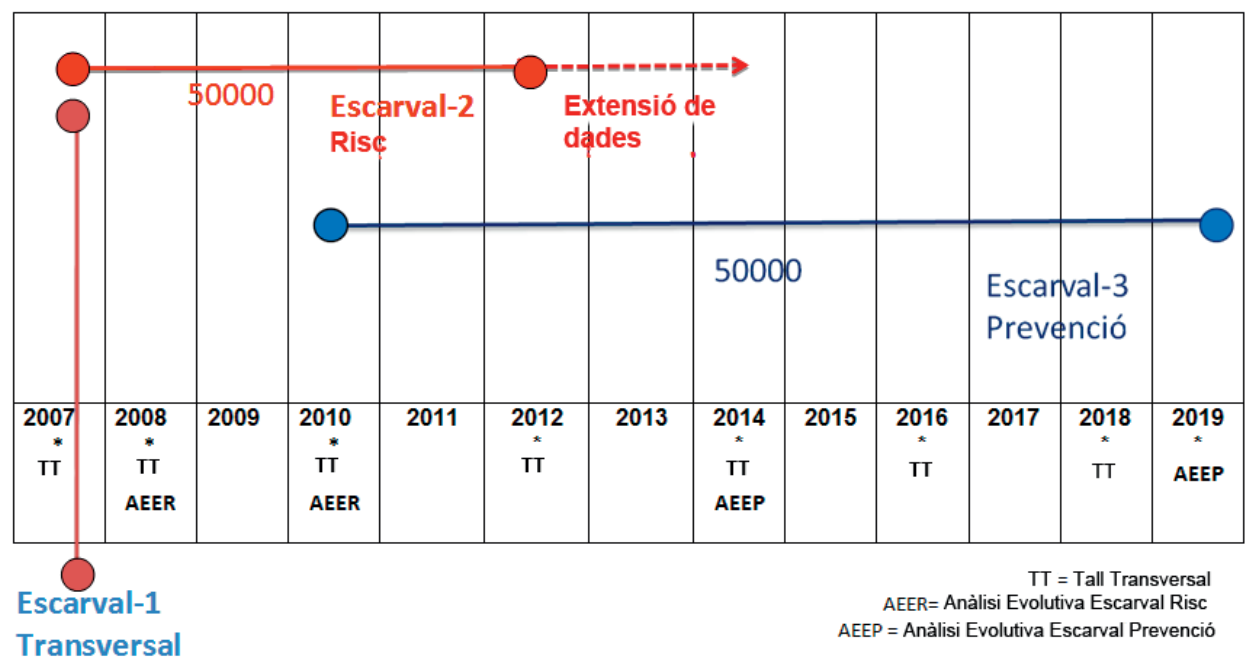

Esquema 1. Plasmació cronològica del desenvolupament dels tres projectes d'investigació ESCARVAL.

\section{BIBLIOGRAFIA}

Aranceta, J. i altres (2004): «Grupo colaborativo para el estudio Dorica fase II. Tablas de evaluación del riesgo coronario adaptadas a la población española. Estudio DORICA», Medicina Clínica, 123, 686-691.

BANEGAS, J.R. i altres (2003): «Mortality attributable to cardiovascular risk factors in Spain», European Journal of Clinical Nutrition, 57, 18-21.

BANEgas, J.R. i altres (2011): Estudio de Nutrición y Riesgo Cardiovascular en España (ENRICA), Madrid, Universidad Autónoma de Madrid.

Brotons, C. i altres (2004): «Adaptación española de la Guía Europea de Prevención Cardiovascular. Comité Español Interdisciplinario para la Prevención Cardiovascular (CEIPC)», Atención Primaria, 34, 427-432.

Califf, R.M. i R. Platt (2013): «Embedding Cardiovascular Research Into Practice», The Journal of the American Medical Association, 310 (19), 2037-2038.

Collins, G.S. i D.G. Altman (2012): «Predicting the 10 year risk of cardiovascular disease in the United Kingdom: independent and external validation of an updated version of QRISK2», BMJ, 344, e4181.

ConRoy, R.M. i altres (2003): «Estimation of ten-year risk of fatal cardiovascular disease in Europe: the SCORE project», European Heart Journal, 24, 987-1003.

D'Agostino, R.B. i altres (2001): «Validation of the Framingham Coronary Hearth Disease Prediction Scores: results of a multiple ethnic groups investigation», The Journal of the American Medical Association, 286, 180-187.

De BACKer, G. i altres (2003): «Executive summary. European guidelines on cardiovascular disease prevention in clinical practice», European Heart Journal, 24, 1601-1610.

Folsom, A.R. (2013): «Classical and novel biomarkers for cardiovascular risk prediction in the United States», Journal of Epidemiology, abril, 23, 158-162.

Greenland, P. i altres (2010): «ACCF/AHA guideline for assessment of cardiovascular risk in asymptomatic adults: Executive summary. A report of the American College of Cardiology Foundation/American Heart Association Task Force on Practice Guidelines», Circulation, 122, 2748-2764. 
Grundy, S.M. i altres (2014): «Expert Dyslipidemia Panel of the International Atherosclerosis Society Panel members. An International Atherosclerosis Society Position Paper: global recommendations for the management of dyslipidemia», Journal of Clinical Lipidology, 8, 29-60.

Guallar-Castillón, P. i altres (2012): «Magnitude and management of hypercholesterolemia in the adult population of Spain, 2008-2010: The ENRICA Study», Revista Española de Cardiología, juny, 65(6), 551-558.

Health Quality Ontario (2013): «Electronic tools for health information exchange: an evidence-based analysis», Ontario Health Technology Assessment Series, setembre, 13 (11), 1-76.

HENSE, H.W. i altres (2003): «Framingham risk function overestimates risk of coronary hearth disease in men and women from Germany: results of the MONICA Augsburg and the PROCAM cohorts», European Heart Journal, 24, 937-945.

HEss, C.N. i altres (2013): «Embedding a randomized clinical trial into an ongoing registry infrastructure: unique opportunities for efficiency in design of the Study of Access site For Enhancement of Percutaneous Coronary Intervention for Women (SAFE-PCI for Women)», American Heart Journal, $166,421-428$.

Institut Nacional D’Estadística (2012): Mortalidad cardiovascular en España, Madrid, Gobierno de España, <http://www.ine.es/jaxi/menu.do?type=pcaxis \&path=\%2Ft20\%2Fe306\&file=inebas $\mathrm{e} \& \mathrm{~L}=0>$.

KuUlasmaA, K. i altres (2000): «Estimation of contribution of changes in classic risk factors to trends in coronary-event rates across the WHO MONICA Project populations», Lancet, 355, 675-687.

Lloyd-Jones, D.M. i altres (2010): «American Heart Association Strategic Planning Task Force and Statistics Committee. Defining and setting national goals for cardiovascular health promotion and disease reduction: the American Heart Association's Strategic Impact Goal through 2020 and beyond», Circulation, 121, 586-613.

MAIQUes, A. i altres (2014) «Recomendaciones preventivas cardiovasculares en atención primaria. Actualización 2012 del Programa de Actividades Preventivas y de Promoción de la Salud (PAPPS)», Atención Primaria, juny, 46 (4), 3-15.

MARrugat, J. i altres (2003a): «An adaptation of the Framingham risk function to southern Europe Mediterranean areas», Journal of Epidemiology \& Community Health, 57, 634-638.

MARrugat, J. i altres (2003b): «Estimación del riesgo coronario en España mediante la ecuación de Framingham calibrada», Revista Española de Cardiología, 56, 253-261.

MASIÁ, R. i altres (1998): «High prevalence of cardiovascular risk factors in Girona, Spain, a province with low myocardial infarction incidence», Journal of Epidemiology \& Community Health, 52, 707-715.

MEdRANo, M.J. i altres (2007): «Coronary disease risk attributable to cardiovascular risk factors in the Spanish population», Revista Española de Cardiología, desembre, 60, 1250-1956.

Menotti, A. i altres (2000a): «Heart disease incidence in Northern and Southern Europeans populations: a reanalysis of the seven countries study for an European coronary risk chart», Heart, 84, 238-44.

Menotti, A. i altres (2000b): «Coronary Heart disease incidence in Northern and Southern European populations: a reanalysis of the seven countries study for an European coronary risk chart», Heart, $84,238-44$.

Menotti, A. i altres (2000c): «Comparison of the Framingham risk function-based coronary chart risk function from an Italian population study», European Heart Journal, 21, 365-370.

Pérez, G. i altres (1998): «Acute myocardial infarction case fatality, incidence and mortality rates in a population registry in Girona, Spain, 1990-1992», International Journal of Epidemiology, 27, 599-604.

Rojo-Martínez, G. i altres (2013): «Use of Drugs Related to the Treatment of Diabetes Mellitus and Other Cardiovascular Risk Factors in the Spanish Population. The Di@bet.es Study», Revista Española de Cardiología, 66, 854-863.

Rose, G. (1985): «Sick individuals and sick populations», International Journal of Epidemiology, 14, 32-38.

Sociedad Española de Hipertensión (2005): Guía Española de Hipertensión, Madrid, SEH-LELHA, $<$ http://www.seh-lelha.org/guiahta05.htm>. 
Soriguer, F. i altres (2012): «Prevalence of diabetes mellitus and impaired glucose regulation in Spain: the Di@bet.es Study», Diabetologia, 55, 88-93.

TomÁs, L. i altres (2001): «Factores de riesgo y morbimortalidad coronaria en una cohorte laboral mediterránea seguida durante 28 años. Estudio Manresa», Revista Española de Cardiología, 54, 1146-1154.

WiLson, P.W. i altres (1998): «Prediction of coronary hearth disease using risk factor categories», Circulation, 97, 1837-1847.

\section{BIONOTES}

\section{Domingo Orozco}

MD, PhD. Especialista en Medicina de família. President de la Comissió Nacional de l'especialitat de Medicina de Família Co-coordinador de l'estratègia d'abordatge de la Cronicitat del Ministeri de Sanitat, Política Social i Igualtat. Professor associat del Departament de Medicina Clínica de la Universitat Miguel Hernández.

\section{Vicente Pallarés}

MD PHD. Especialista en Medicina Familiar i Comunitària. Especialista Universitari en Gestió de la Cronicitat. Clinical Hypertension Specialists per l'European Society of Hypertension. Responsable del Sistema de Gestió de Seguretat del Pacient, Unidad de Vigilància de la Salut. Unión de Mutuas, Castelló. Professor associat del Departament de Medicina de la Universitat Jaume I. Professor col-laborador en el Màster d'Atenció Primària del Departament de Medicina de la Universita Miguel Hernández i professor col-laborador honorífic de la mateixa universitat. Membre del cos docent de la Cátedra de Cronicidad SEMERGEN-Universidad Europea de Madrid i València. President de la Societat Valenciana d'Hipertensió i Risc Vascular.

\section{Vicente Gil Guillén}

MD, PhD. Especialista en Medicina de Família. Director de la Càtedra de Medicina Clínica, Facultat de Medicina, Universitat Miguel Hernández. Director del Màster d'Atenció Primària de la Universitat Miguel Hernández. Membre del Comitè Científic del Projecte Escarval. 